\title{
Post-Processing of Fabry-Pérot Microcavity Tip Sensor
}

\author{
Marta S. Ferreira, Joerg Bierlich, S. Unger, Kay Schuster, José Luís Santos, and Orlando Frazão
}

\begin{abstract}
A Fabry-Pérot microcavity tip sensor fabricated by post-processing of a special design double-cladding optical fiber is proposed. The produced fiber has a pure silica core, an outer cladding, and an inner silica cladding surrounding the core doped with phosphorous. When subjected to chemical etching postprocessing, the whole ring region is removed and light is guided in the core region. The sensing head is created by splicing this fiber to single mode fiber and applying chemical etching to the fiber end. The core is forming a tip and it is thus surrounded by air. The Fabry-Pérot microcavity tip sensor is subjected to temperature, and a sensitivity of $15.5 \mathrm{pm} /{ }^{\circ} \mathrm{C}$ is obtained.
\end{abstract}

Index Terms-Double-clad optical fiber, post-processing, wet chemical etching.

\section{INTRODUCTION}

$\mathbf{T}$ HE usage of sensors in extreme temperature conditions is of vital importance for many industries, such as car, aerospace, military, oil, among others. The real time monitoring of this parameter is requested in spite of the harsh conditions. Optical fiber sensors are very suitable to be used in such conditions provided their robustness and ability to achieve high sensitivities.

Fiber optic sensors based on Fabry-Pérot (FP) cavities have been widely explored over the last decades [1]. One way to obtain the cavity consists on modifying the fiber itself through post-processing. In 2007, a FP etalon fabricated in a photonic crystal fiber by using a $157 \mathrm{~nm}$ laser was presented [2]. The etalon, insensitive to temperature, was used to measure strain at different temperatures. Fabrication of FP cavities using femtosecond laser have also been reported, where micronotches [3] and microchannels [4] have been subjected to temperature and refractive index, respectively. A different technique consists on creating a microscopic air bubble when splicing two fibers [5]-[7]. In such case, the reported sensors presented low thermal sensitivity. Finally, the etching post-processing can give rise to different topologies.

Manuscript received May 9, 2013; revised June 7, 2013; accepted June 19, 2013. Date of current version July 29, 2013. This work was supported in part by the COST Action under Grant TD1001 and in part by the Fundação para a Ciência e Tecnologia under Grant SFRH / BD / 76965 / 2011.

M. S. Ferreira and J. L. Santos are with the INESC Porto, Porto 4169-007, Portugal, and also with the Faculdade de Ciências da Universidade do Porto, Porto 4169-007, Portugal (e-mail: msaf@inescporto.pt; josantos@fc.up.pt).

J. Bierlich, S. Unger, and K. Schuster are with the IPHT, Jena - Institute of Photonic Technology, Jena 07745, Germany (e-mail: joerg.bierlich@ ipht-jena.de; sonja.unger@ipht-jena.de; kay.schuster@ipht-jena.de).

O. Frazão is with the INESC Porto, Porto 4169-007, Portugal (e-mail: ofrazao@inescporto.pt).

Color versions of one or more of the figures in this letter are available online at http://ieeexplore.ieee.org.

Digital Object Identifier 10.1109/LPT.2013.2271325
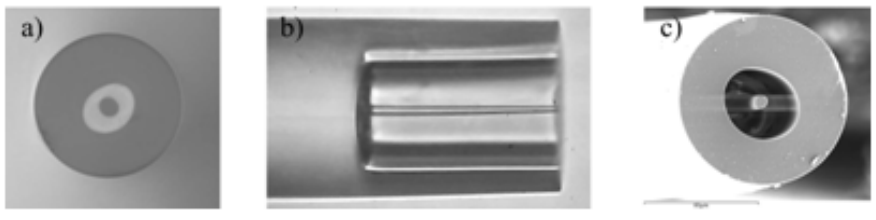

Fig. 1. Phosphorous-doped double-clad optical fiber photographs a) prior to etching post-processing; b) after etching post-processing, longitudinal view; and c) SEM top image after etching.

In 2007, a study of the etching behavior of silica fiber sensors using hydrofluoric (HF) acid, as well as their response to load was described [8]. In 2011, a FP strain sensor obtained by etching a fiber with a specific geometry and composition was reported [9]. The same group presented an FP cavity composed by one in-fiber-mirror, whilst the other was created by an air cavity with diaphragm [10]. Furthermore, two FP interferometers based on chemical etching of multimode graded index fibers were investigated [11]. The sensors were used to perform simultaneous measurement of strain and temperature.

Usually, double-clad fibers are used either to control the fiber dispersion or to generate high power lasers. In this letter, due to the post-processing, a new application is proposed. A specially designed to be post-processed double-clad optical fiber is presented. Initially, there is no light guidance in the central core as the high refractive index of the inner cladding ensures that all light is deviated from the core. However, after etching the inner cladding will be removed and light will be guided in the suspended core. A FabryPérot cavity is created and the sensor is tested for temperature variations.

\section{OPTICAL FibER TIP FORMATION}

The phosphorous-doped double-clad optical fiber (DCF) shown in Fig. 1, whose preform was obtained by modified chemical vapor deposition method, was designed to have specific dimensions after wet etching post-processing. These dimensions assure the possibility of creating an interferometer that can be used as a sensing element. Thus, both core and outer cladding were constituted by pure silica and had initial diameter dimensions of $18 \mu \mathrm{m}$ and $122 \mu \mathrm{m}$, respectively. In between these two components, there was a $15 \mu \mathrm{m}$ thick layer of P-doped silica glass, which formed the inner cladding. This layer had a phosphorous concentration of $11 \mathrm{~mol}-\%$. Initially, light will travel in the inner cladding region instead of the core, since its refractive index is higher than the 


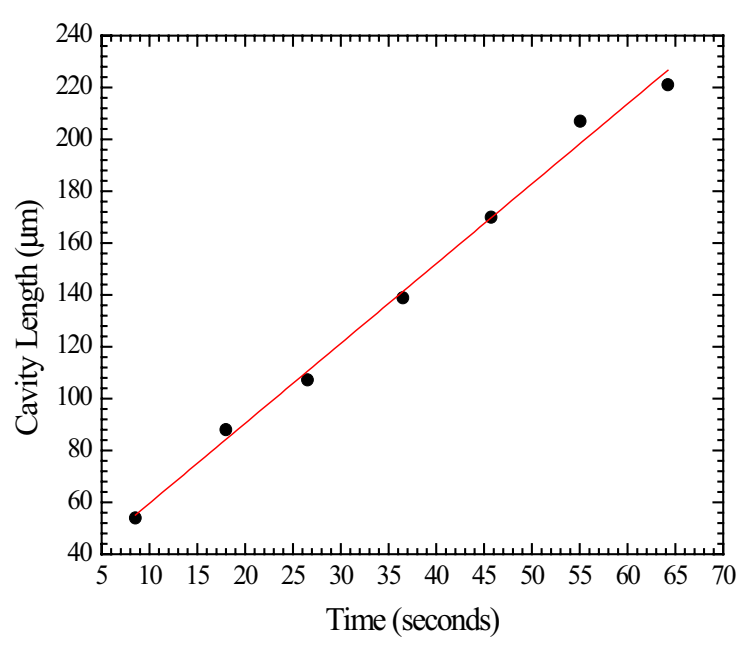

Fig. 2. Cavity formation with time.

one of the core ( $\Delta n$ was determined to be, approximately $9.5 \times 10^{-3}$ higher in the P-doped region than in the nondoped ones). When subjected to wet etching post-processing, the refractive index in the core becomes higher than the one of the surrounding medium (air). In its turn, the light will be guided through the core. The optical fiber tips were produced by splicing a section of a few hundred micrometers long P-doped DCF to single mode fiber. The splices were carried out with the automatic program of the splice machine. Afterwards, the tip was immersed in a solution of hydrofluoric acid (HF) with $48 \%$ concentration, at room temperature $\left(\sim 22{ }^{\circ} \mathrm{C}\right)$. In order to increase the etching rate, necessary to obtain a nearly constant core diameter over the fiber tip, the HF solution was placed inside an ultrasound bath. The chemical reactions involved in this process were [8], [12]:

$$
\begin{gathered}
\mathrm{SiO}_{2}+6 \mathrm{HF} \rightarrow \mathrm{H}_{2} \mathrm{Si} \mathrm{F}_{6}+2 \mathrm{H}_{2} \mathrm{O} \\
\mathrm{P}_{2} \mathrm{O}_{5}+6 \mathrm{HF} \rightarrow 2 \mathrm{POF}_{3}+3 \mathrm{H}_{2} \mathrm{O} .
\end{gathered}
$$

Due to the presence of phosphorus inside the inner cladding, this region will be etched faster than the non doped layers, accordingly to the study presented in [12], [13]. Thus, a microcavity is formed as can be seen in Fig. 1 b). Fig. 1 c) shows the SEM top image of the sensing head after post-processing. The etching behavior is shown in Fig. 2. The linear dependence of the cavity length with time leads to an etching rate of $\sim 3.1 \mu \mathrm{m} / \mathrm{s}$.

\section{EXPERIMENTAL RESULTS}

The experimental setup, exhibited in Fig. 3, was constituted by a broadband optical source with a bandwidth of $100 \mathrm{~nm}$ and centered at $1570 \mathrm{~nm}$, an optical circulator and an optical spectrum analyzer used to measure the reflection signal with a resolution of $0.5 \mathrm{~nm}$. The cavity used to perform the measurements had a length of $\sim 130 \mu \mathrm{m}$ and a core diameter at the end of the tip of $\sim 6 \mu \mathrm{m}$. In the inset of Fig. 3, a microscope photography of the sensing head can be seen. The sensing head was illuminated with a He-Ne laser and the two reflection mirrors of the FP cavity can be distinguished, one in the splice

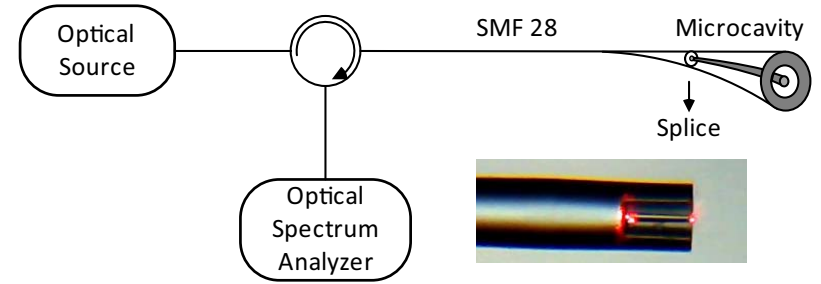

Fig. 3. Experimental setup. Inset: microscope photography when the sensing head was illuminated with a He-Ne laser.

between SMF28 and the DCF, and the other one at the end of the DCF tip. The sensing head spectrum is exhibited in Fig. 4. The observed interference can be approximated to a two-wave FP interferometer. Even though the core region presents large dimension ( $\sim 6 \mu \mathrm{m}$ diameter), the spectral response does not present a multimodal interference. This can be due to the excitation being done through singlemode fiber and to the small cavity length, which is not long enough to excite a large number of modes.

It has been reported in the literature the optimum coupling between a SMF and a silica rod occurs for a silica rod diameter of $\sim 16 \mu \mathrm{m}$ (in this situation the Fresnel reflection at the interface is minimum) [17], close to the actual $18 \mu \mathrm{m}$ diameter of the double-clad optical fiber. In the present configuration this characteristic would not be desirable because such Fresnel reflection generates one of the waves of the low-finesse FP interferometer. It is avoided in view the etching process that reduces the DCF core diameter to $6 \mu \mathrm{m}$, value that introduces a substantial mismatch with the effective diameter of the SMF guided mode, generating in this way an effective Fresnel reflection.

A decrease of the optical power with wavelength is observed in the Figure, which is due to the sensing head losses. A simple theoretical model based on the one presented by Rao et al. [14] was used to simulate this FP cavity spectrum. In this case, only two mirrors are considered: one at the SMF28-DCF interface and the other at the DCF-air interface. Therefore, the reflection coefficients are given by:

$$
R_{1}=\left(\frac{n_{S M F}-n_{D C F}}{n_{S M F}+n_{D C F}}\right)^{2} \quad, \quad R_{2}=\left(\frac{n_{D C F}-n_{\text {air }}}{n_{D C F}+n_{\text {air }}}\right)^{2}
$$

where $n_{S M F}, n_{D C F}$ and $n_{\text {air }}$ are the refractive indices of each medium. Notice that $n_{D C F}=1.43$ corresponds to the effective refractive index of the DCF, which was calculated through the experimental spectrum. The round-trip propagation shift is given by $\Phi=4 \pi n_{D C F} L / \lambda$, where $L$ is the cavity length and $\lambda$ is the wavelength. Considering that there is a high transmission loss due to mode mismatch between the two fibers, resulting into a transmission coefficient of $(1-\alpha)$ with $\alpha=0.01$, the total reflective intensity can be obtained through eq. 4.

$$
\begin{aligned}
I_{\text {Rtotal }}(\lambda)= & R_{1}+(1-\alpha)^{2}\left(1-R_{1}\right)^{2} R_{2} \\
& +2 \sqrt{R_{1} R_{2}}(1-\alpha)\left(1-R_{1}\right) \cos (2 \Phi) .
\end{aligned}
$$




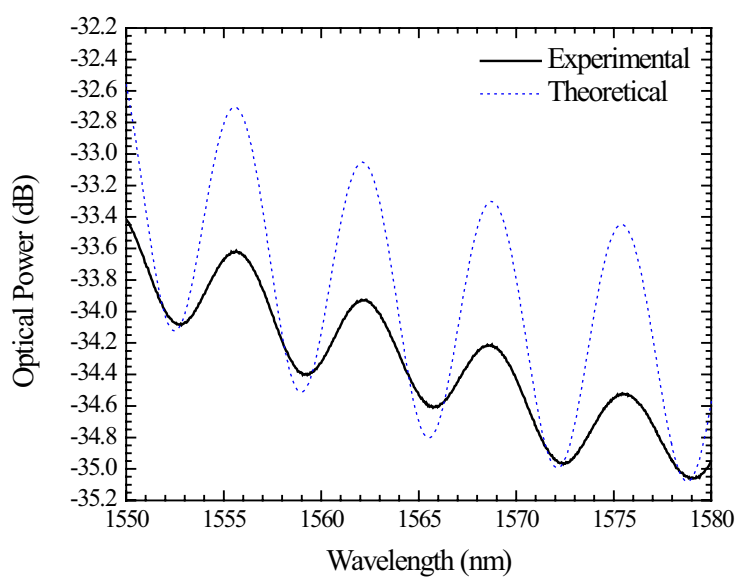

Fig. 4. Experimental (black line) and theoretical (blue dotted line) spectra of the sensing head reflection response.

The total optical power calculated through the theoretical model is shown in Fig. 4 (the blue dotted line), along with the experimental spectrum (black line). The observed wavelength dependence is similar in both cases, in the theoretical results the average optical power is roughly $0.5 \mathrm{~dB}$ higher compared with the experimental ones. The large difference appears in the visibility of the interferometric fringes, resulting substantially higher in the analysis, a consequence of not considering coherence effects (the geometric path imbalance of the cavity, $2 \times 130 \mu \mathrm{m}$, is substantially higher than the coherence length of the optical source). For the temperature measurements, the sensing head was placed inside a tubular oven and the readings were done with a resolution of $0.1{ }^{\circ} \mathrm{C}$. This sensing head proved to be suitable for a wide range of temperatures, from $150{ }^{\circ} \mathrm{C}$ to $900{ }^{\circ} \mathrm{C}$, as can be observed in Fig. 5. There is a spectrum red shift as temperature increases, which means that the thermal expansion is the dominant effect. Furthermore, the sensing head behavior is quite similar for the measurements done with increasing and decreasing temperature. The temperature range can be divided in two groups, low and high temperatures. According to table I, for lower temperatures, the rms error is slightly higher than for higher ones. However, the correlation factor $\left(r^{2}\right)$ is very high in both cases. Besides, the sensitivity is larger for high temperatures. These facts indicate that the linear fit is suitable for this sensor in the $150{ }^{\circ} \mathrm{C}-900{ }^{\circ} \mathrm{C}$ range, where a sensitivity of $14.6 \mathrm{pm} /{ }^{\circ} \mathrm{C}$ was obtained. This value is higher than the typical one for a fiber Bragg grating [15] $\left(11 \mathrm{pm} /{ }^{\circ} \mathrm{C}\right.$ at $\left.1550 \mathrm{~nm}\right)$. It should also be highlighted that this sensor presents a sensitivity $\sim 60 \%$ higher than the one obtained for a hollow core silica tube tested with the same configuration [16]. The equivalent coefficient of thermal expansion (CTE) of the device was calculated to be $8 \times 10^{-5} /{ }^{\circ} \mathrm{C}$, which is higher than the known CTE of core the fused silica, $5.5 \times 10^{-7} /{ }^{\circ} \mathrm{C}$. The thermally induced bending of the device, partially evidenced by modification of the spectral response when the sensor is subjected to high temperatures, mainly caused the difference of the large device CTE.

\section{CONCLUSION}

In summary, a double-clad optical fiber temperature sensor was proposed. The new fiber presented an inner cladding

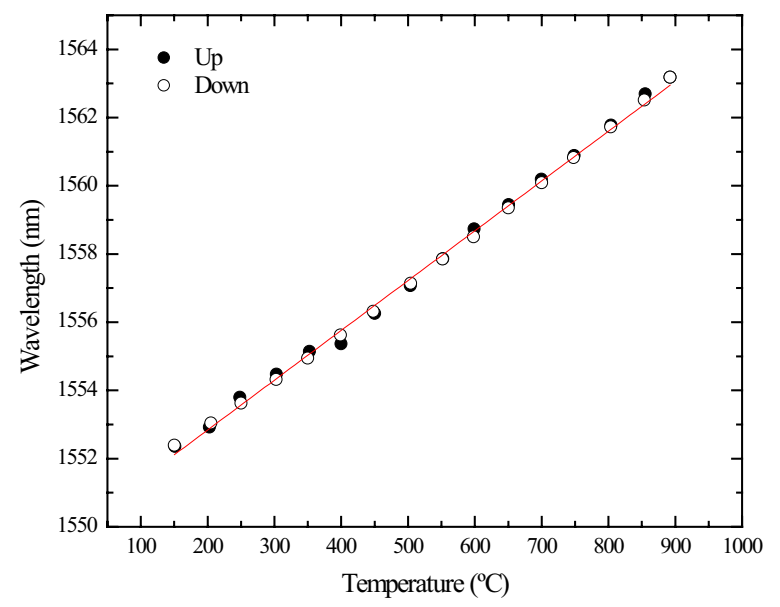

Fig. 5. Fringe pattern wavelength shift with the applied temperature.

TABLE I

SENSITIVITY AND RMS ERRORS FOR DIFFERENT TEMPERATURE RANGES

\begin{tabular}{cccc}
\hline \hline $\begin{array}{c}\text { Temperature } \\
\text { range }\left({ }^{\circ} \mathrm{C}\right)\end{array}$ & $\begin{array}{c}\text { Sensitivity } \\
\left(\mathrm{pm} /{ }^{\circ} \mathrm{C}\right)\end{array}$ & $r m s\left(\mathrm{pm} /{ }^{\circ} \mathrm{C}\right)$ & $r^{2}$ \\
\hline $150-900$ & 14.60 & 0.15 & 0.99835 \\
$150-450$ & 13.22 & 0.15 & 0.99921 \\
$500-900$ & 15.51 & 0.12 & 0.99953 \\
\hline \hline
\end{tabular}

composed by phosphorous-doped silica, with a higher refractive index than the pure silica core. By applying postprocessing, the light pathway was altered and the fiber started to guide light inside the core. When the sensing head was subjected to a temperature range of $750{ }^{\circ} \mathrm{C}$, a linear sensitivity of $14.6 \mathrm{pm} /{ }^{\circ} \mathrm{C}$ was achieved. However, when used to measure high temperatures, a sensitivity of $15.5 \mathrm{pm} /{ }^{\circ} \mathrm{C}$ was obtained. Thus, this sensing head can be a suitable candidate to perform real-time monitoring of extreme temperatures.

\section{REFERENCES}

[1] T. Zhu, D. Wue, M. Liue, and D.-W. Duane, "In-line fiber optic interferometric sensors in single-mode fibers," Sensors, vol. 12, no. 8, pp. 10430-10449, Aug. 2012.

[2] Z. L. Ran, Y. J. Rao, H. Y. Deng, and X. Liao, "Miniature in-line photonic crystal fiber etalon fabricated by $157 \mathrm{~nm}$ laser micromachining," Opt. Lett., vol. 32, pp. 3071-3073, Nov. 2007.

[3] T. Wei, Y. Han, H.-L. Tsai, and H. Xiao, "Miniaturized fiber inline Fabry-Perot interferometer fabricated with a femtosecond laser," Opt. Lett., vol. 33, pp. 536-538, Mar. 2008.

[4] K. Zhou, Z. Yan, L. Zhang, and I. Bennion, "Refractometer based on fiber Bragg grating Fabry-Pérot cavity embedded with a narrow microchannel," Opt. Express, vol. 19, pp. 11769-11779, Jun. 2011.

[5] J. Villatoro, V. Finazzi, G. Coviello, and V. Pruneri, "Photonic-crystalfiber-enabled micro-Fabry-Perot interferometer," Opt. Lett., vol. 34, pp. 2441-2443, Aug. 2009.

[6] F. C. Favero, L. Araujo, G. Bouwmans, V. Finazzi, J. Villatoro, and V. Pruneri, "Spheroidal Fabry-Perot microcavities in optical fibers for high-sensitivity sensing," Opt. Express, vol. 20, pp. 7112-7118, Mar. 2012.

[7] T. Wang, M. Wang, and H. Ni, "Micro-Fabry-Pérot interferometer with high contrast based on an in-fiber ellipsoidal cavity," IEEE Photon. Technol. Lett., vol. 24, no. 11, pp. 948-950, Jun. 2012.

[8] V. R. Machavaram, R. A. Badcock, and C. F. Fernando, "Fabrication of intrinsic fibre Fabry-Perot sensors in silica fibres using hydrofluoric acid etching," Sens. Actuators A, Phys., vol. 138, pp. 248-260, Jul. 2007.

[9] S. Pevec and D. Donlagic, "All-fiber, long-active-length Fabry-Perot strain sensor," Opt. Express, vol. 19, pp. 15641-15651, Aug. 2011. 
[10] S. Pevec and D. Donlagic, "Miniature all-fiber Fabry-Perot sensor for simultaneous measurement of pressure and temperature," Appl. Opt., vol. 51, pp. 4536-4541, Jul. 2012.

[11] P. A. R. Tafulo, P. A. S. Jorge, J. L. Santos, F. M. Araujo, and O. Frazao, "Intrinsic Fabry-Pérot cavity sensor based on etched multimode graded index fiber for strain and temperature measurement," IEEE Sensors J., vol. 12, no. 1, pp. 8-12, Jan. 2012.

[12] J. Bierlich, J. Kobelke, D. Brand, K. Kirsch, J. Dellith, and H. Bartelt, "Nanoscopic tip sensors fabricated by gas phase etching of optical glass fibers," Photon. Sensors, vol. 2, pp. 331-339, Jul. 2012.

[13] S. Pevec, E. Cibula, B. Lenardic, and D. Donlagic, "Micromachining of optical fibers using selective etching based on phosphorus pentoxide doping," IEEE Photon. J., vol. 3, no. 4, pp. 627-632, Aug. 2011.
[14] Y.-J. Rao, M. Deng, D.-W. Duan, and T. Zhu, "In-line fiber FabryPerot refractive-index tip sensor based on endlessly photonic crystal fiber," Sens. Actuators A, Phys., vol. 148, no. 1, pp. 33-38, Nov. 2008.

[15] Y.-J. Rao, "In-fibre Bragg grating sensors," Meas. Sci. Technol., vol. 8, no. 4, pp. 355-375, Nov. 1997.

[16] M. S. Ferreira, L. Coelho, K. Schuster, J. Kobelke, J. L. Santos, and O. Frazão, "Fabry-Perot cavity based on a diaphragm-free hollow-core silica tube," Opt. Lett., vol. 36, pp. 4029-4031, Oct. 2011.

[17] S. Pevec and D. Donlagic, "Miniature micro-wire based optical fiber-field access device," Opt. Express, vol. 20, pp. 27874-27887, Dec. 2012. 